\title{
Improving epidemics control strategies by extended detection
}

\author{
Paweł Karp, ${ }^{1, *}$ Bartłomiej Dybiec, ${ }^{1, \dagger}$ and Adam Kleczkowski ${ }^{2}, \ddagger$ \\ ${ }^{1}$ Marian Smoluchowski Institute of Physics, and Mark Kac Center for Complex Systems Research, \\ Jagiellonian University, ul. Reymonta 4, 30-059 Kraków, Poland \\ ${ }^{2}$ Dept. Computing Science and Mathematics, University of Stirling, Stirling FK9 4LA, United Kingdom
}

(Dated: September 20, 2013)

\begin{abstract}
Majority of epidemics eradication programs work in preventive responsive way. The lack of exact information about epidemiological status of individuals makes responsive actions less efficient. Here, we demonstrate that additional tests can significantly increase the efficiency of "blind" treatment (vaccination or culling). Eradication strategy consisting of "blind" treatment in very limited local neighbourhood supplemented by extra tests in a little bit larger neighbourhood is able to prevent invasion of even highly infectious diseases and to achieve this at a cost lower than for the "blind" strategy. The effectiveness of the extended strategy depends on such parameters as the test efficiency and test cost.
\end{abstract}

PACS numbers: $05.40 . \mathrm{Fb}, 82.20 . \mathrm{Wt}$, 02.50.-r, 02.50.Ey, 87.19.Xx.

Keywords: epidemiological modelling, disease spread, stochastic modelling, epidemiological control

\section{INTRODUCTION}

Many outbreaks of serious diseases of humans (e.g. SARS [1]), animals (e.g. foot-and-mouth disease [2]), and plants epidemics (e.g. citrus canker [3] and sudden oak death [4]) can be described in terms of a spread of an infectious agent on a network of hosts. This representation can then successfully be used to design control strategies [5, 6, 18-27]. However, two factors limit the use of modelling for real systems. Firstly, the topology of the network is usually not completely known as we do not possess a full information on which nodes are in contact with which. Secondly, the status of individuals, whether they are susceptible, infected, infectious or removed/recovered, is often not known. We have shown [5-7] that despite these drawbacks it is possible to design control strategies optimal in a combined epidemiological and economic sense.

Any successful control strategy for infectious diseases needs to balance at least two conflicting goals. On one hand, it aims at a quick eradication of the disease agent. Thus, from purely epidemiological point of view, the best approach would be to remove all the cases (sources of infection) immediately. At the same time, due to limited resources and time constraints, the epidemic has to be controlled at manageable costs within realistic time window. The solution is to find a mixture of preventive and responsive local or global treatments $[5,6,8]$. Potential treatments can include vaccination, culling, or even isolation (quarantine) of individuals.

In this paper we consider an epidemic that spreads on a network with local nearest-neighbour interactions. Control measures which are triggered by certain events are

\footnotetext{
*Electronic address: pawel.karp@uj.edu.pl

$\dagger$ Electronic address: bartek@th.if.uj.edu.pl

$\ddagger$ Electronic address: adamk@mathbio.com
}

applied locally within a fixed range. However, in extension to previous work, we also consider further diagnostic tests which are performed on all agents located within a test range. The positive results of diagnostic tests lead to further treatment (within the same fixed radius as the initial control action) centered on diagnosed individuals. Consequently, the suggested strategy is a mixture of a local "blind" strategy $[9,10]$ and "live test" strategy; the latter originally proposed to attempt to eradicate bovine tuberculosis [11-13]. The designers of the strategy involving tests claim that it leads to a lower number of badgers killed than the "clean ring" strategy in which all badgers are killed in a certain radius from a focus of infection. The "clean ring" strategy usually results in unnecessary killing of healthy individuals and the use of diagnostic tests shifts the standard responsive (event triggered) "blind" control strategy to the proactive regime. Thus, application of diagnostic tests makes the eradication strategy more selective in comparison to the clean ring strategy, which extends the control radius until ring of uninfected individuals is removed.

In our paper we firstly present the model and define the cost associated with the control strategy. We assume that control strategy is purely local, however, it is supplemented by additional diagnostic tests. We subsequently study the sensitivity of the cost to the choice of the control radius as defined in $[6,9,10]$, of the test radius and of the test sensitivity. We show that under certain conditions additional testing can improve efficiency of local control strategies in comparison to purely local methods.

\section{MODEL AND RESULTS}

The model extends the basic SIR (susceptible - infectious - recovered) epidemiological model [14, 15] by introduction of two additional classes: detected (infectious symptomatic) and vaccinated/treated [5]. Our paper is focused on the optimization of control strategies 


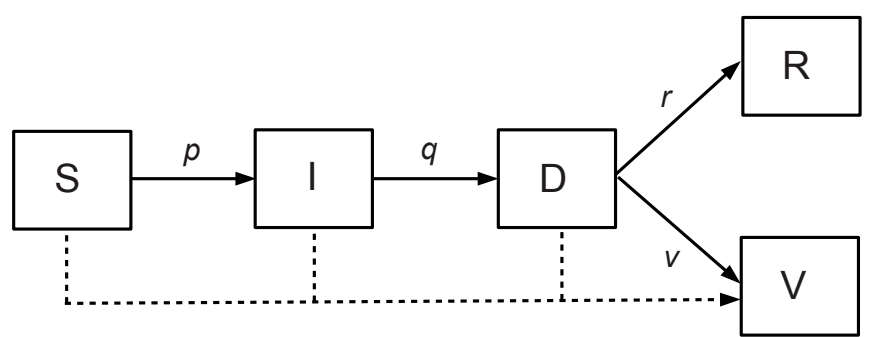

FIG. 1: The diagram showing possible states of individuals and allowed transitions. Labels assigned to links represent probabilities of given transitions. Transitions to the infectious state (I) can be induced by contact with any infectious individual (I or D). During a treatment not only the detected individual is vaccinated but also its nearest neighbours up to the order $z_{\mathrm{v}}$, what is schematically indicated by the dashed line.

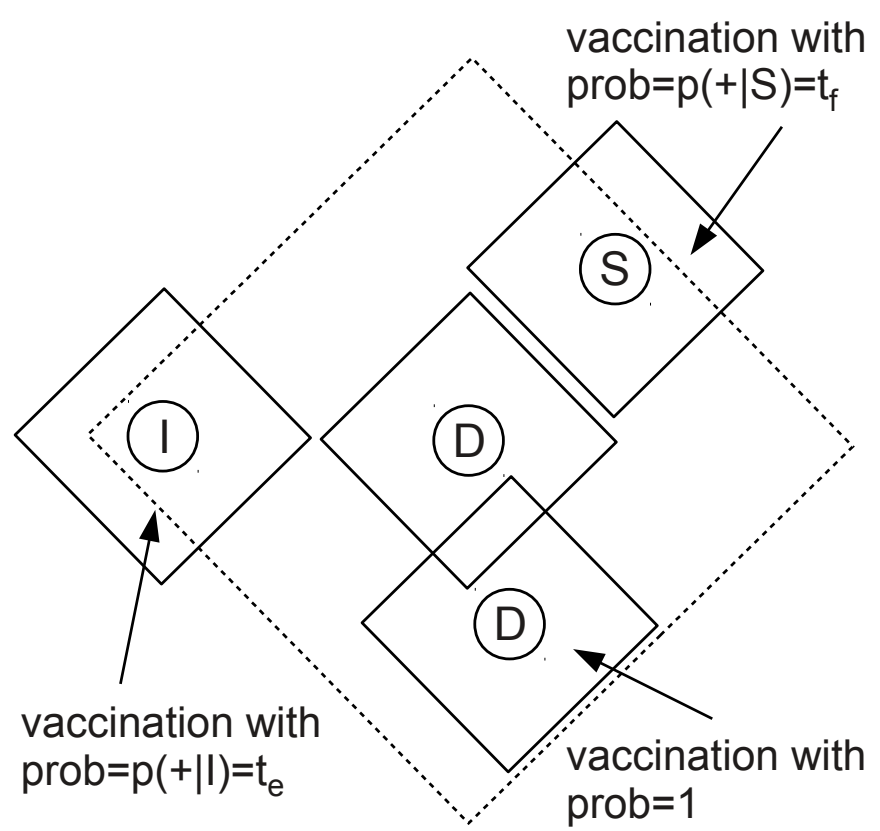

FIG. 2: Schematic representation of the extended control strategy: solid lines represent treatment neighbourhoods $\left(z_{\mathrm{v}}\right)$ while dashed line the test neighbourhood $\left(z_{\mathrm{t}}\right)$. An detected individual $D$ can trigger a control measure with probability $v$. If a control measure is applied, every detected $D$ individual in the test neighbourhood $\left(z_{\mathrm{t}}\right)$ triggers a treatment of all its local neighbours up to $z_{\mathrm{v}}$. Also a susceptible individual or infectious individual can trigger a treatment with probabilities $p(+\mid S)=t_{\mathrm{f}}$ and $p(+\mid S)=t_{\mathrm{e}}$ respectively.

when exact epidemiological status of each individual is unknown - in particular when only detected individuals can be observed by public health authorities. In this paper we consider an additional testing procedure that can be used to distinguish between different classes of individuals. However, this procedure is associated with additional costs and is not perfect.

\section{A. Model}

The system consist of $N$ individuals placed on a regular square lattice with periodic boundary conditions. Every individual can be in one of the following states

S - susceptible,

I - infected pre-symptomatic,

D - infected symptomatic (detected),

$\mathbf{R}$ - recovered,

V - vaccinated.

Possible transitions between states are presented in Fig. 1. There is no return transition from the vaccinated or recovered state to the susceptible state, i.e. treatment or recovery result in perfect, permanent immunity (from the mathematical point of view vaccinated and recovered states are absorbing). Any susceptible individual can be infected due to a contact with an infectious (infected or detected) individual, with probability $p$ per contact. Infectious individuals start to display symptoms after a spontaneous transition to the detected class, with probability $q$; subsequently they can recover with probability $r$. In addition, every detected individual can trigger a control event with probability $v$. This results in a series of actions taken by the health authorities in order to stop the disease from spreading.

Basic strategy: In the simplest case, the control action will consist of treatment of the detected individual together with all its local neighbours up to the order $z_{\mathrm{v}}$ (basic strategy). This strategy corresponds to a "blind" strategy as it treats all individuals in the neighbourhood regardless of their status, and it broadly corresponds to measures used in controlling the foot-and-mouth [2] or citrus canker [3] diseases. In order to compensate for the lack of knowledge of exact epidemiological status of individuals, the treatment is performed in a larger neighbourhood of the detected individual $z_{\mathrm{v}}$ in hope of catching all potentially infected but presymptomatic individuals $\left(z_{\mathrm{v}}=0\right.$ correspond to treatment of the detected individual only).

Extended strategy: The basic strategy assumes that we only know the location of detected individuals, when they exhibit visible symptoms. However, in some cases it might be possible to improve the detectability by introduction of additional testing. As before, every infectious symptomatic individual (D) triggers a control measure with probability $v$, but this time the control event consists of two steps:

1. The detected individual together with (all) its nearest neighbours up to the $z_{\mathrm{v}}$ order are vaccinated.

2. In addition to step 1 , state dependent actions are performed on all local neighbours up to the order $z_{\mathrm{t}}$ (test range) of the detected individual, as follows 
$\mathbf{S}$ - additional test is performed,

$\mathbf{I}$ - additional test is performed,

D - rule 1 is performed and individuals and their neighbours are treated,

$\mathbf{R}$ - no additional action is taken,

V - no additional action is taken.

If result of the test is positive, rule 1 is applied on the "positive" recognised individuals (which are then treated together with their neighbours up to the $z_{\mathrm{v}}$ order). Otherwise, no further actions are taken.

The rules listed above assume that we are able to "visually" distinguish between $\mathrm{R}$ and $\mathrm{V}$ classes on one hand (the individuals might know that they have recovered from the disease or have been treated; alternatively a record might exist that proves their status) and $\mathrm{S}$ and I classes on the other hand. It is only the latter two classes that are tested to determine their exact status and in particular to detect individuals in the I class. We do not specify the details of the test; presumably this can be done by immunological methods similar to the "live test" procedure for bovine tuberculosis in badgers [11-13].

The test is characterized by three parameters: $t_{\mathrm{e}}-$ the probability of detection of infectious individual given it is actually infectious, i.e. $p(+\mid I)$, and $t_{\mathrm{f}}-$ the probability of false detection, i.e. $p(+\mid S)$ (we will only study $t_{\mathrm{e}}$ in this paper and assume that $t_{\mathrm{f}}$ is fixed). In addition, each test has a price $t_{\text {price }}$. The extended strategy is presented schematically in Fig. 2. The control action was initiated by the detected individual located in the centre. First, all individuals within the small (solid lines) diamond are treated. Second, all individuals within the larger (broken lines) diamond are tested. All individuals in class D trigger further action (diamond centred around the second individual D). All individuals that test diagnosed positively (whether they in reality are $\mathrm{S}$ or I) also trigger further treatment events (two diamonds centred around individuals $\mathrm{S}$ and $\mathrm{I}$ ). The treatment range $z_{\mathrm{v}}$ and test range $z_{\mathrm{t}}$ are two independent parameters, however, the most efficient control strategy corresponds to $z_{\mathrm{t}}>z_{\mathrm{v}}$ with low $z_{\mathrm{v}}$, see below.

The main aim of the paper is to compare efficiency of the basic strategy (with fixed $z_{\mathrm{v}}$ ) with the extended strategy (with the same $z_{\mathrm{v}}$ and varying $z_{\mathrm{t}}$ ). The comparison is done by using the overall cost associated with the eradication strategy is $X=V+R$ (basic strategy) or $X=V+R+t_{\text {price }} \times N_{T}$ (extended strategy), where $V, R$ and $N_{T}$ represent the number of vaccinated individuals, recovered individuals and the total number of performed test respectively.

\section{B. Results}

The model described in Sec. A has been studied by simulations. First, for the reference, the results for the basic eradication strategy are presented. Next, it is shown how and when the extended strategy can outperform the basic control strategy.

The simulations have been performed for $r=0.01$ (recovery probability), $v=0.5$ (treatment probability), $t_{\mathrm{f}}=$ $0.05=p(+\mid S)$ (probability of false detection), $p=2^{p_{\exp }}$ (infection probability) and $q=0.2$ (probability of symptoms occurrence). The test range is $z_{\mathrm{t}}=\{0,1,2,3\}$, the treatment range is $z_{\mathrm{v}}=\{1,2,3,4\}$, however we concentrate on the results for $z_{\mathrm{v}}=1$. This corresponds to the worst-case scenario for the basic strategy. Thus, if testing can indeed improve the overall performance, this will be easier to assess for $z_{\mathrm{v}}=1$ than for $z_{\mathrm{v}}>1$.

\section{Basic strategy - no testing}

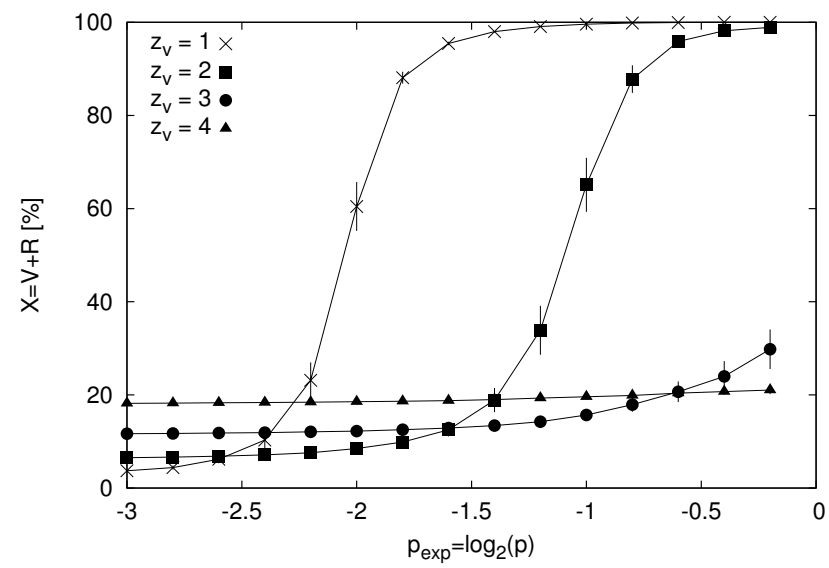

FIG. 3: Cost of the basic control strategy $X\left(z_{\mathrm{t}}=0\right)$ for various treatment ranges: $z_{\mathrm{v}}=\{1,2,3,4\}$. Other simulation parameters $q=0.2, r=0.01, v=0.5$. Results have been averaged over 100 realizations. System size is $200 \times 200$. Error bars represent standard deviation of the mean.

Figure. 3 presents results for the basic strategy, i.e. strategy without tests. Here, the cost of control strategy $X$ is defined as $X=V+R$, where $V$ and $R$ are numbers of vaccinated and recovered individuals respectively, i.e. it is the number of individuals affected (directly or indirectly) by the epidemic outbreak. The total cost $X$ increases as the infection probability $p$ increases, for all values of the control neighbourhood, $z_{\mathrm{v}}$. Rapid increase in $X$ for a certain value of $p$ corresponds to a switch from non-invasive, fully controlled epidemic to an invasive one which is no longer controlled. We do not show the results for $R$ and $V$ separately, but simulations show that in this case the cost of control strategy is dominated by treat- 
ment, i.e. epidemic runs out of control and it is necessary to treat the whole population. The main effect of changing $z_{\mathrm{v}}$ is to shift the critical value of $p$ towards higher probabilities. This means that the higher $z_{\mathrm{v}}$ is, any outbreak of the more infectious disease can successfully be stopped by application of such a strategy.

However, for a fixed $p$ the relationship between $X$ and $z_{\mathrm{v}}$ is non-trivial. For very small $p$, smaller treatment range $\left(z_{\mathrm{v}}\right)$ generates smaller overall cost of eradication strategy. As $p$ increases, the strategy with $z_{\mathrm{v}}=1$ becomes insufficient and the number of vaccinated individuals and consequently the overall cost $X$ increase rapidly (crosses in Fig. 3). As a result $z_{\mathrm{v}}=2$ leads to the lowest value of $X$ and so can be interpreted as an optimal strategy. This process is repeated for higher values of $p$, as $z_{\mathrm{v}}=3$ and subsequently $z_{\mathrm{v}}=4$ become optimal. For parameters considered here $z_{\mathrm{v}}=4$ stays optimal even for $p$ close to 1 .

\section{Extended strategy}

For the extended control strategy, the total cost of the epidemic includes an additional component due to testing, i.e. $X=V+R+t_{\text {price }} \times N_{T}$, where $t_{\text {price }}$ is the price of a single test while $N_{T}$ is the total number of tests performed. In order to explore the effect of testing, we assume the worst case scenario for the basic strategy, $z_{\mathrm{v}}=1$. We also initially assume that the test is not very efficient, $t_{\mathrm{e}}=60 \%$, see Fig. 4 and later which explore the dependence of the results on $t_{\mathrm{e}}$.

The increase in the infectivity is now compensated not by the increase in the treatment range $z_{\mathrm{v}}$ (as for the basic strategy, see Fig. 3), but by introduction of testing in the neighbourhood of size $z_{\mathrm{t}}$ which varies from $z_{\mathrm{t}}=0$ (no testing, see Fig. 3 ) to $z_{\mathrm{t}}=3$. For $z_{\mathrm{t}}=0$ and $z_{\mathrm{t}}=1$, the dependence on $p$ is characterised by the threshold behaviour, with no invasion for small values of $p$ and invasion followed by treatment of almost whole population for large $p$ (compare Fig. 3 with Fig. 4). However, for $z_{\mathrm{t}}=1$ the threshold value of $p$ is shifted towards much higher values with only minimal increase in overall costs for small $p$. Thus, although $z_{\mathrm{t}}=0$ is optimal for subcritical epidemics (small $p$ ), the addition of testing significantly improves the efficiency of the control in a large range of $p$ (up to $p \simeq 0.43$, see Fig. 4). Increasing the radius of testing neighbourhood from $z_{\mathrm{t}}=1$ to $z_{\mathrm{t}}=2$ increases the overall cost, $X$, for small to medium values of $p$, but it removes the threshold behaviour entirely. Testing with $z_{\mathrm{t}}=1$ still leads to the smallest value of $X$ for $p \lesssim 0.43$ at which point $z_{\mathrm{t}}=2$ becomes optimal. Further increase in the testing effort (to $z_{\mathrm{t}}=3$ ) consistently increases the costs in the whole range of $p$ values. We therefore conclude that for this combination of other parameters, $z_{\mathrm{t}}=0$ is optimal for very small values of $p$ (where we would not have treated anyway), $z_{\mathrm{t}}=1$ is optimal for $p \lesssim 0.43$, and $z_{\mathrm{t}}=2$ is optimal afterwards. Thus, even if the test is relatively inefficient, $t_{\mathrm{e}}=60 \%$, it
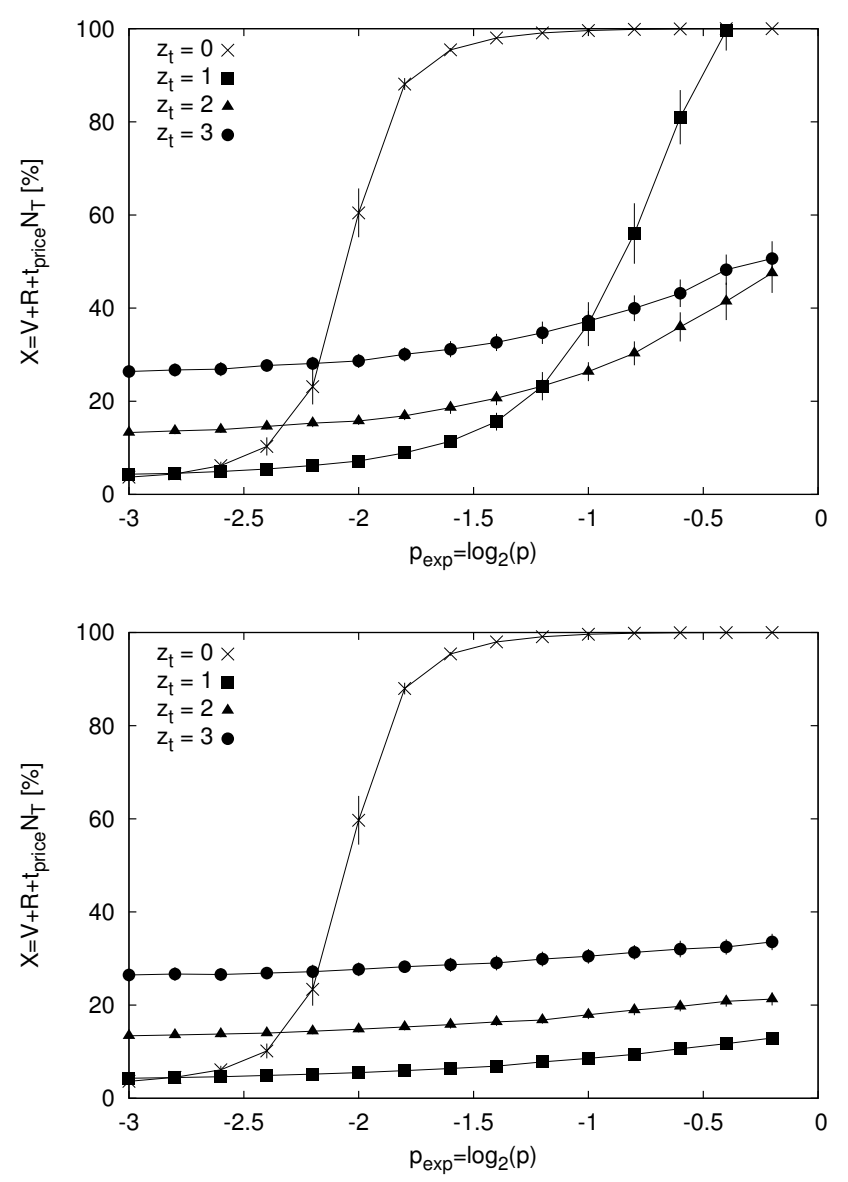

FIG. 4: Cost of the extended control strategy $X$ as a function of the probability of spread, $p$, for different values of $z_{\mathrm{t}}$, with $z_{\mathrm{t}}=0$ corresponding to no testing; top panel: low test efficiency, $t_{\mathrm{e}}=0.6$, bottom panel: high test efficiency, $t_{\mathrm{e}}=0.9$. Other parameters: $z_{\mathrm{v}}=1, t_{\text {price }}=0.25, q=0.2, r=0.01$, $v=0.5$. Results have been averaged over 100 realizations. System size is $200 \times 200$. Error bars represent standard deviation of the mean.

is always preferable to combine "blind" treatment with testing, providing the cost is low $\left(t_{\text {price }}=0.25\right)$.

Increasing the efficiency of testing from $t_{\mathrm{e}}=0.6$ (meaning that $40 \%$ of infectious individuals are misdiagnosed as non-infectious) to $t_{\mathrm{e}}=0.9$ makes $z_{\mathrm{t}}=1$ the optimal solution even for very high values of $p$, see the bottom panel of Fig. 4. There is no threshold behaviour for $z_{\mathrm{t}}>0$ and so epidemics are controlled even for very high values of $p$.

Returning to $t_{\mathrm{e}}=0.6$ we now study the effect of changing the test price, $t_{\text {price }}$, on the choice of the control strategy. Decrease in the cost of testing does not change the picture qualitatively; the main effect is the overall reduction in the total cost, $X$, compare top panel of Fig. 4 with Fig. 5. The reduction in $t_{\text {price }}$ also reduces values of the infection probability $p$ at which the optimal strategy changes from $z_{\mathrm{t}}=1$ to $z_{\mathrm{t}}=2$ (from $p \simeq 0.43$ for 


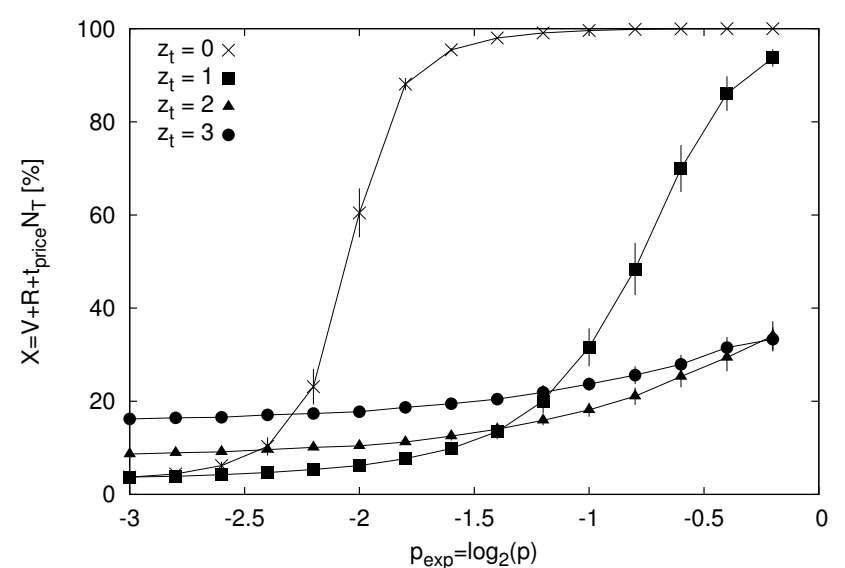

FIG. 5: Similar to Fig. 4, but for the lower test cost, $t_{\text {price }}=$ 0.05 per test. Other parameters: $z_{\mathrm{v}}=1, t_{\mathrm{e}}=0.6, q=0.2$, $r=0.01, v=0.5$. Results have been averaged over 100 realizations. System size is $200 \times 200$. Error bars represent standard deviation of the mean.

$t_{\text {price }}=0.25$ to $p \simeq 0.38$ for $t_{\text {price }}=0.05$ ).

Finally, we study a combined effect of changing $\left(z_{\mathrm{v}}, z_{\mathrm{t}}\right)$ on the choice of the optimal strategy. In Fig. 6 we have shown the dependence of both test range $z_{\mathrm{t}}$ (individual curves) and treatment range $z_{\mathrm{v}}$ (individual panels). There is very little qualitative effect of changing $z_{\mathrm{v}}$ on the dependence of $X$ on $z_{\mathrm{t}}$; the strategy with $z_{\mathrm{t}}=1$ is always the best of the three, even though the actual values of $X$ vary. There is also no threshold behaviour with respect to $p$ for $z_{\mathrm{t}}>0$; addition of testing always stops any epidemic considered here.

However, comparison of the results with $z_{\mathrm{t}}=0$ and $z_{\mathrm{t}}=1$ for $z_{\mathrm{v}}>1$ reveal an interesting result. Although for $z_{\mathrm{v}}=1$ the strategy without testing $\left(z_{\mathrm{t}}=0\right)$ is almost always less efficient than with testing $\left(z_{\mathrm{t}}=1\right)$, this changes when $z_{\mathrm{v}}$ is increased. As treatment radius $z_{\mathrm{v}}$ increases, the range of the infection probability $p$ for which $z_{\mathrm{t}}=0$ is optimal is enlarged. Putting it differently, for large $z_{\mathrm{v}}$ the "blind" control strategy (basic strategy) can outperform the extended strategy (strategy with the test). Nevertheless, even for $z_{\mathrm{v}}=3$ there is still a considerable interval of $p$ values for which the extended strategy is better than the basic strategy, see bottom panel of Fig. 6.

\section{SUMMARY AND CONCLUSIONS}

Efficient eradication of epidemics relies on quick and efficient control measures. The lack of knowledge of exact epidemiological status of each individual makes responsive actions more complicated and potentially less efficient. One possible solution to this problem is to increase the treatment range in order to compensate for the lack of knowledge about the status of individuals.
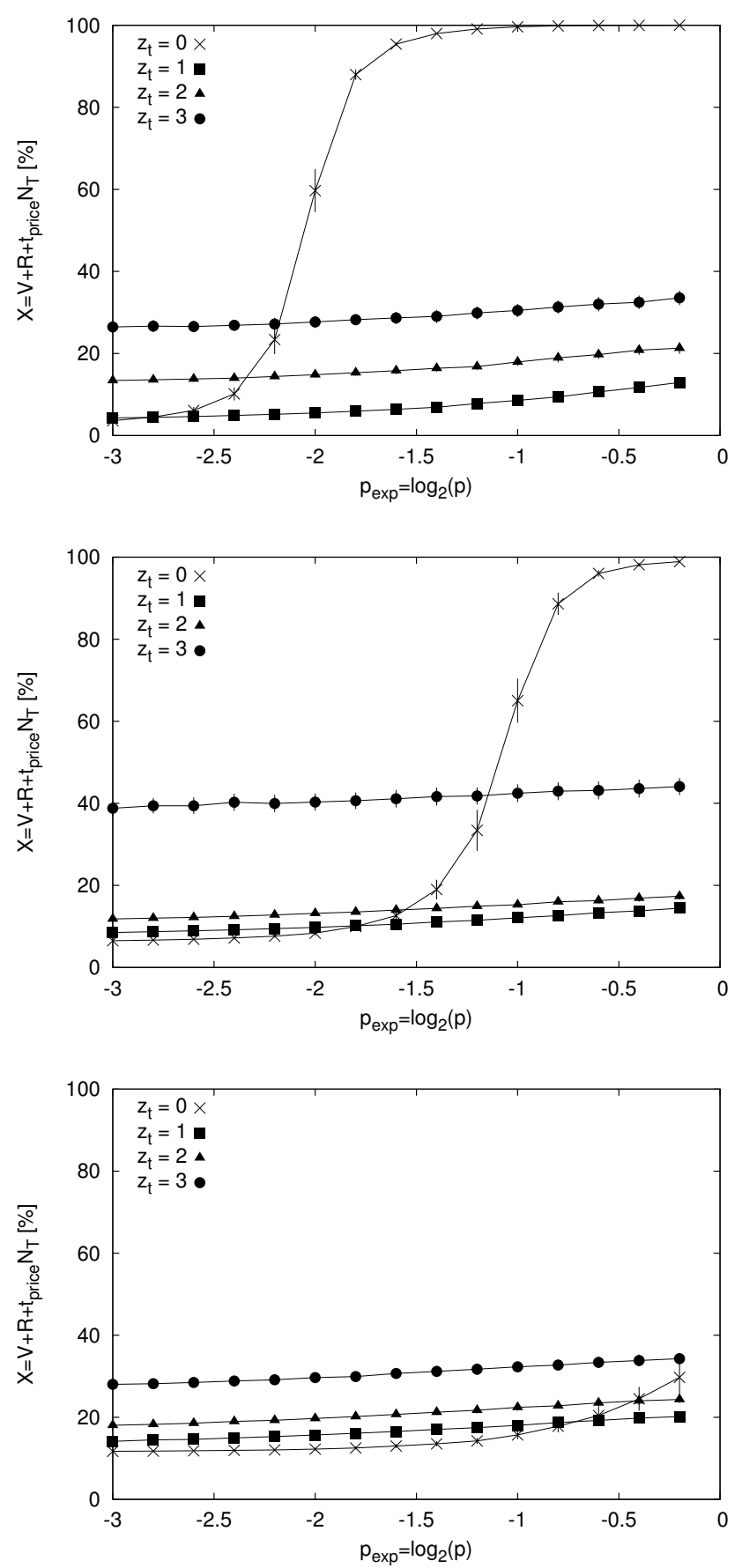

FIG. 6: Similar to Fig. 4 (bottom panel), but for different values of $z_{\mathrm{v}}$, top panel: $z_{\mathrm{v}}=1$ (identical to bottom panel of Fig. 4, but shown here for completeness), middle panel: $z_{\mathrm{v}}=2$, bottom panel: $z_{\mathrm{v}}=3$. Other parameters: $t_{\mathrm{e}}=0.9$, $t_{\text {price }}=0.25, q=0.2, r=0.01, v=0.5$. Results have been averaged over 100 realizations. System size is $200 \times 200$. Error bars represent standard deviation of the mean.

Alternative solution considered in this paper is based on additional tests which can improve early detection of in- 
fectious pre-symptomatic individuals.

Our basic strategy assumes "blind" treatment of all local neighbours within a given radius. Thus, it could lead to unnecessary waste of resources which are used for treatment of healthy individuals especially in situations when treatment range is large. This is the general drawback of the "clean ring" strategy considered in the context of bovine tuberculosis control [11-13]. Extended strategy as proposed here acts in a more selective (proactive) way since an initial control event is followed by responsive actions driven by the epidemiological status of individuals. Consequently, "blind" treatment in small neighbourhood supplemented by tests in a little bit larger neighbourhood result in lower overall cost of eradication of epidemic than the "blind" treatment. One of our key results is to show that complementing the "blind" strategy with a minimal treatment range $\left(z_{\mathrm{v}}=1\right)$ by testing with a minimal radius $\left(z_{\mathrm{t}}=1\right)$ followed by another round of treatment is sufficient to stop outbreaks of an even highly infectious disease. We also show that high test efficiency is crucial for high overall performance of the control strategy. This result is analogous to the one for the "live test" strategy [11-13].

The extended strategy avoids several disadvantages of the "clean ring" strategy because "blind" treatment is performed in the limited range only. The control area is extended in the selective (proactive) way by the diagnostic tests which are performed on the all (potentially infec- tious) individuals within the test range. This results in a relative large number of tested individuals, in turn improving relatively poor statistics of the "live test" strategy [11-13]. Consequently, combined action of "blind" treatment (in small neighbourhood) and diagnostic test improves the efficiency of local "blind" (basic) control strategy.

Our work can be extended in a number of ways. Firstly, optimisation in our case has only been performed with respect to one variable. We first fix treatment range $z_{\mathrm{v}}$ and then look for optimisation with respect to the test range $z_{\mathrm{t}}$ (with sensitivity analysis to study the dependence of the results on the test efficiency $t_{\mathrm{e}}$ and test price $\left.t_{\text {price }}\right)$. In reality, the policy maker can optimise with respect to both $z_{\mathrm{v}}$ and $z_{\mathrm{t}}$, exploring trade offs between treatment and testing. Secondly, our results assume that treatment costs are the same as infection costs. Thus, in a more general formula for $X=c V+R$ we have assumed $c=1$. This assumption can be relaxed, see $[6,7]$. Thirdly, we only considered selected values of $t_{\mathrm{e}}$ and $t_{\text {price }}$ and a more systematic sensitivity analysis remains to be done. Fourthly, we only studied one parameter characterising the test efficiency, $t_{\mathrm{e}}$; the remaining parameter describing the probability of false positives has been fixed. Finally, we used a very simple network; more complicated networks have been considered in this context $[5,6,28-36]$.
[1] C. Dye and N. Gay, Science 300, 1884 (2003).

[2] M. J. Keeling et al., Science 294, 813 (2001).

[3] T. R. Gottwald et al., Phytopathology 91, 30 (2001).

[4] D. M. Rizzo et al., Plant Disease 86, 205 (2002).

[5] B. Dybiec, A. Kleczkowski, and C. A. Gilligan, Phys. Rev. E 70, 066145 (2004).

[6] A. Kleczkowski, K. Oleś, E. Gudowska-Nowak, and C. A. Gilligan, J. R. Soc. Interface 9, 158 (2012).

[7] K. Oleś, E. Gudowska-Nowak, and A. Kleczkowski, PloS one 7, e36026 (2012).

[8] G. A. Forster and C. A. Gilligan, Proc. Natl. Acad. Sci. U.S.A. 104, 4984 (2007).

[9] B. Dybiec, A. Kleczkowski, and C. A. Gilligan, Acta Phys. Pol. B 36, 1509 (2005).

[10] A. Kleczkowski, B. Dybiec, and C. A. Gilligan, Acta Phys. Pol. B 37, 3017 (2006).

[11] G. C. Smith, C. L. Cheeseman, D. Wilkinson, and R. S. Clifton-Hadley, J. Appl. Ecol. 38, 520 (2001).

[12] G. C. Smith, C. L. Cheeseman, R. S. Clifton-Hadley, and D. Wilkinson, J. Appl. Ecol. 38, 509 (2001).

[13] R. Woodroffe, S. D. W. Frost, and R. S. Clifton-Hadley, J. Appl. Ecol. 36, 494 (1999).

[14] R. M. Anderson and R. M. May, Infectious diseases of humans: Dynamics and control (Oxford University Press, Oxford, 1991).

[15] H. W. Hethcote, SIAM Rev. 42, 599 (2000).

[16] B. Dybiec, A. Kleczkowski, and C. A. Gilligan, J. R. Soc. Interface 6, 941 (2009).

[17] K. Oleś, E. Gudowska-Nowak, and A. Kleczkowski, PloS one 8, e63813 (2013).

[18] L. A. Meyers, M. E. J. Newman, M. Martin, and S. Schrag, Emerging Infectious Diseases 9, 204 (2003).

[19] M. J. Keeling and P. Rohani, Modeling Infectious Diseases in Humans and Animals (Princeton University Press, ADDRESS, 2007), p. 408.

[20] A. L. Hill, D. G. Rand, M. a. Nowak, and N. a. Christakis, PLoS computational biology 6, e1000968 (2010).

[21] D. M. Green, I. Z. Kiss, and R. R. Kao, Proceedings. Biological sciences / The Royal Society 273, 2729 (2006).

[22] Y. Maeno, Physica A: Statistical Mechanics and its Applications 389, 4755 (2010).

[23] M. E. J. Newman, Physical Review E 66, (2002).

[24] M. J. Keeling, Proceedings. Biological sciences / The Royal Society 266, 859 (1999).

[25] F. Iozzi et al., PLoS computational biology 6, e1001021 (2010).

[26] J. Miller and J. Hyman, Physica A: Statistical Mechanics and its Applications 386, 780 (2007).

[27] S. Eubank et al., Nature 429, 180 (2004).

[28] C. Christensen, I. Albert, B. Grenfell, and R. Albert, Physica A 389, 2663 (2010).

[29] C. Barrett, S. Eubank, and V. Kumar, SIAM news 37, 1 (2004).

[30] B. Wang, L. Cao, H. Suzuki, and K. Aihara, Journal of Physics A: Mathematical and Theoretical 44, 035101 (2011).

[31] J. H. Jones and M. Salathe, PLoS Computational Biology 6, e1000736 (2010). 
[32] R. Pastor-Satorras and A. Vespignani, Physical Review Letters 86, 3200 (2001).

[33] I. Z. Kiss, D. M. Green, and R. R. Kao, Proceedings. Biological sciences / The Royal Society 272, 1407 (2005).

[34] S. Riley, Science (New York, N.Y.) 316, 1298 (2007).
[35] N. Ferguson, Nature 446, 2007 (2007).

[36] C. Moore and M. E. J. Newman, Physical Review E 61, 5678 (2000). 